\title{
Pyomyositis in the setting of complicated diverticulitis: case report
}

This article was published in the following Dove Press journal:

International Journal of General Medicine

James Sun'

David Leor Kashan' Jolita Marie Auguste' Akella Chendrasekhar ${ }^{2}$

'Department of Surgery, State University of New York, Downstate Medical Center, Brooklyn, NY, USA; ${ }^{2}$ Department of Surgery, Richmond University Medical Center, Staten Island, NY, USA
Correspondence: James Sun SUNY Downstate Department of Surgery, 450 Clarkson Ave, Box 40, Brooklyn, NY I I203, USA

$\mathrm{Tel}+\mathrm{I} 7182705800$

Fax + I 7182702826

Email James.Sun@downstate.edu
Abstract: Pyomyositis is typically thought of as a disease of the tropics. However, it is becoming more prevalent in temperate regions, and may be underdiagnosed. Here, pyomyositis is encountered as a complication of perforated diverticulitis, which has not been previously reported. A 61-year-old Caucasian man initially presented in respiratory distress and was diagnosed with respiratory failure due to COPD exacerbation. The patient was taking highdose prednisone, $60 \mathrm{mg}$ daily for the past 2 years. Initially, he was afebrile, normotensive, tachycardic to 178 beats/minute and tachypneic to 28 breaths/minute, requiring noninvasive ventilation to maintain oxygenation. Blood tests revealed leukocytosis of $16.7 \times 10^{3} / \mu \mathrm{L}$, and blood cultures grew Escherichia coli. Broad-spectrum antibiotics were started but leukocytosis and bacteremia persisted on repeated tests. On the seventh hospital day, a CT scan of the abdomen was performed for complaints of abdominal pain, and the patient was diagnosed with Hinchey stage 3 diverticulitis. A Hartmann's procedure was performed with intraoperative findings of purulent peritonitis. Intraoperative cultures grew E. coli and vancomycinresistant Enterococcus faecium. The patient continued to have leukocytosis of $15.1 \times 10^{3} / \mu \mathrm{L}$ despite surgical therapy. He began to complain of left lower extremity pain, and a CT scan on hospital day 24 revealed gluteal intramuscular abscesses, which were percutaneously drained. Persistent symptoms prompted another CT scan on hospital day 28 , which revealed additional intramuscular abscesses in the vastus lateralis muscle, which was also drained, with subsequent resolution of pain and normalization of inflammatory markers. This is the first case demonstrating pyomyositis as a complication of diverticulitis. While the mechanism of pyomyositis may not be unique, it is important to recognize the potential complications of frequently encountered diseases. In this critically ill and immunosuppressed patient, there was delayed diagnosis of both diverticulitis and pyomyositis, but the patient quickly improved once the diseases were recognized and treated.

Keywords: abscess, immunosuppression, steroid use, COPD, intramuscular infection

\section{Introduction}

Pyomyositis is defined as an acute, primary, intramusuclar infection that is hematogenously disseminated from transient bacteremia. ${ }^{1,2}$ This condition was first described by Scriba as a disease of the tropics. ${ }^{1-4}$ It was nearly 100 years before the first reported case in North America by Levin et al and its prevalence has been increasing. ${ }^{5}$ To the author's knowledge, it has only been associated with a surgical disease of the abdomen in a report described by Ea et al as a complication of laparoscopy. ${ }^{6}$ We submit a case of pyomyositis as an adverse outcome of complicated diverticulitis to underscore the importance of this disease as a consideration in a surgical patient. 


\section{Case report}

A 61-year-old Caucasian male initially presented with COPD exacerbation with delirium, visual hallucinations and altered mental status. The patient had been taking high-dose prednisone $60 \mathrm{mg}$ daily for 2 years for COPD. On presentation, the patient was afebrile with a temperature of $98.3^{\circ} \mathrm{F}$, normotensive with a blood pressure of $140 / 85$, tachycardic to 178 beats/minute and tachypneic to 28 breaths/minute, with oxygen saturation of $98 \%$ on bilevel positive airway pressure ventilation. Blood tests were significant for leukocytosis of $16.7 \times 10^{3} / \mu \mathrm{L}$, with a left shift of $89.1 \%$ and a lactic acid of 5.8 $\mathrm{mmol} / \mathrm{L}$. He was admitted to the medical service for respiratory failure secondary to COPD exacerbation. The patient was treated with piperacillin/tazobactam and vancomycin empirically. Leukocytosis improved and blood cultures from admission grew Escherichia coli resistant to ampicillin and levofloxacin, confirmed on repeated blood culture.

On the fifth hospital day, the source of bacteremia was not identified and routine evaluations of the urinary tract and lungs failed to show evidence of infection. The patient had complaints of left flank pain; however, with improved leukocytosis and no other sources of infection, work-up was deferred. On the seventh hospital day, persistent abdominal pain prompted CT imaging of the abdomen, which revealed a $14 \times 3.7 \times 7.2 \mathrm{~cm}$ abscess along the medial wall of the left psoas muscle (Figure 1) and 2 additional abscesses at the L5-S1 level; $5.4 \times 3.6 \mathrm{~cm}$ in the midline and $3.4 \times 2.3 \mathrm{~cm}$ left of midline, and intramuscular air in the left gluteal muscles. The patient was diagnosed with Hinchey stage 3 complicated diverticulitis and the surgery service was consulted. A Hartmann's procedure and abdominal washout was performed, with intraoperative findings of purulent peritonitis.

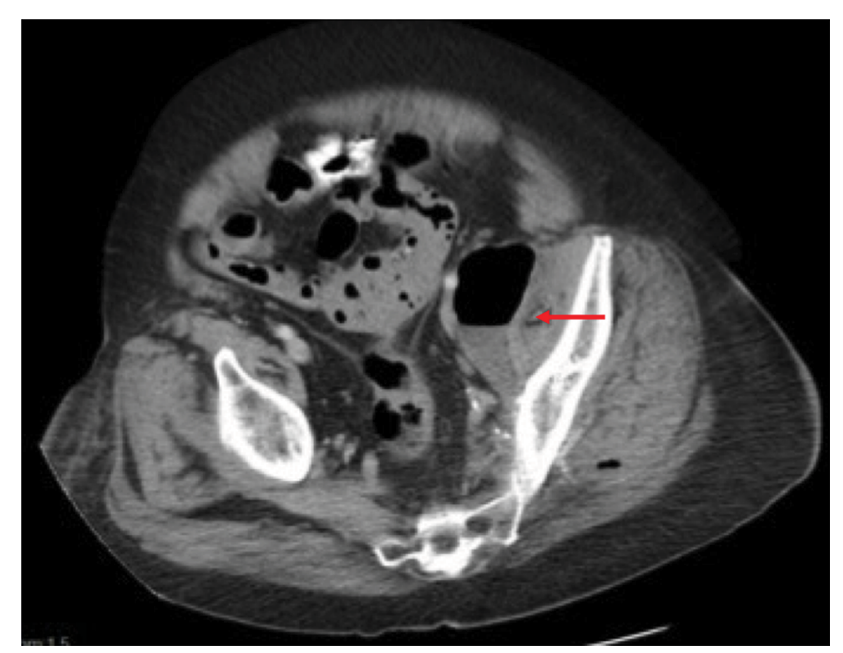

Figure I Initial CT-scan showing abscess with air-fluid level abutting left psoas muscle, indicated by red arrow.
The patient was taken back to the operating room for two additional abdominal washouts during the following week.

Cultures of the peritoneal fluid from the initial procedure revealed E. coli and vancomycin-resistant Enterococcus faecium (VRE). Post-operatively, leukocytosis persisted, reaching a peak of $29.2 \times 10^{3} / \mu \mathrm{L}$ and nadir of $15.1 \times 10^{3} / \mu \mathrm{L}$ despite antibiotic therapy. We subsequently repeated a CTscan on hospital day 24, which was consistent with multiple intramuscular abscesses in the gluteal muscles for which he underwent percutaneous drainage (Figure 2).

Following drainage, leukocytosis remained the same, and the patient began complaining of left lower extremity pain, which was edematous and erythematous on physical examination. After deep venous thrombosis was ruled out, a CT scan was performed on hospital day 28 , which showed a $10 \times 20 \times 3 \mathrm{~cm}$ intramuscular abscess in the vastus lateralis muscle, again treated with percutaneous drainage (Figure 3A-D). Following drainage, cultures were negative, and ultrasound confirmed resolution of abscesses. The patient's symptoms resolved, leukocytosis normalized to $7.7 \times 10^{3} / \mu \mathrm{L}$ and the patient was discharged to a rehabilitation facility on hospital day 34 .

Written informed consent was obtained from the patient and family members to use anonymized information from the medical record, including, but not limited to, images and laboratory values. The patient and family granted permission for the publication of all anonymized information.

\section{Discussion}

Pyomyositis is primarily caused by Staphylococcus aureus, accounting for up to $95 \%$ of reported cases. ${ }^{1-6}$ As mentioned before, it is a primary intramuscular infection, not seeded by penetrating trauma or adjacent soft tissue or bone infections. A plethora of pathogens have been described in prior

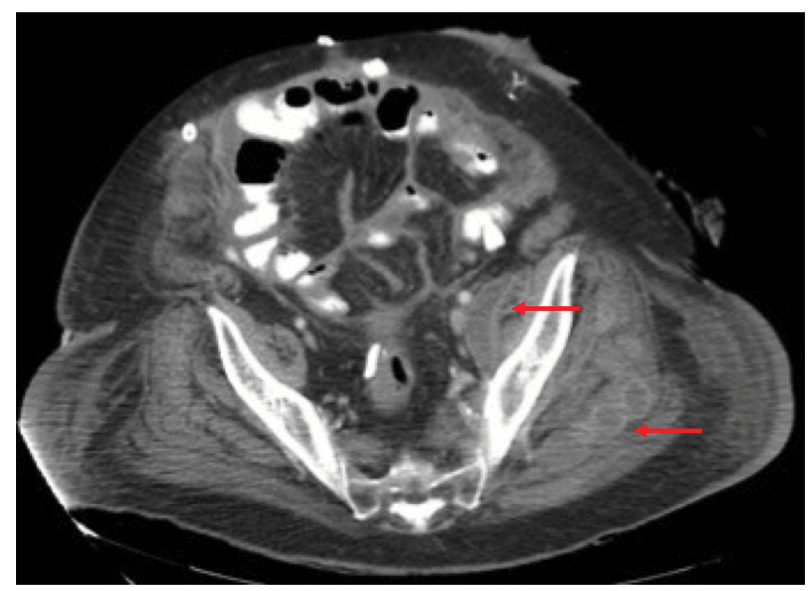

Figure 2 Repeat CT-scan showing abscesses in the left gluteal muscles, indicated by red arrows. 
A

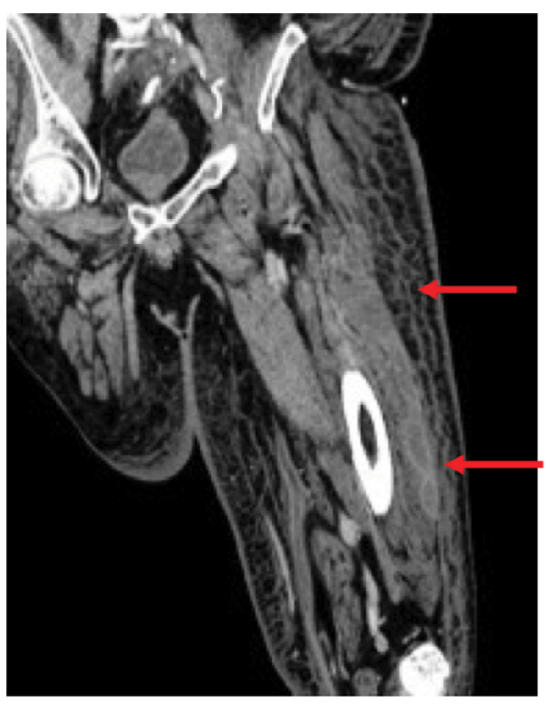

C

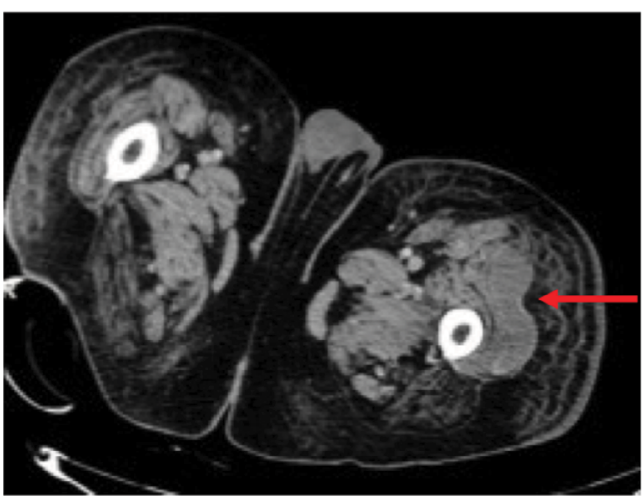

B

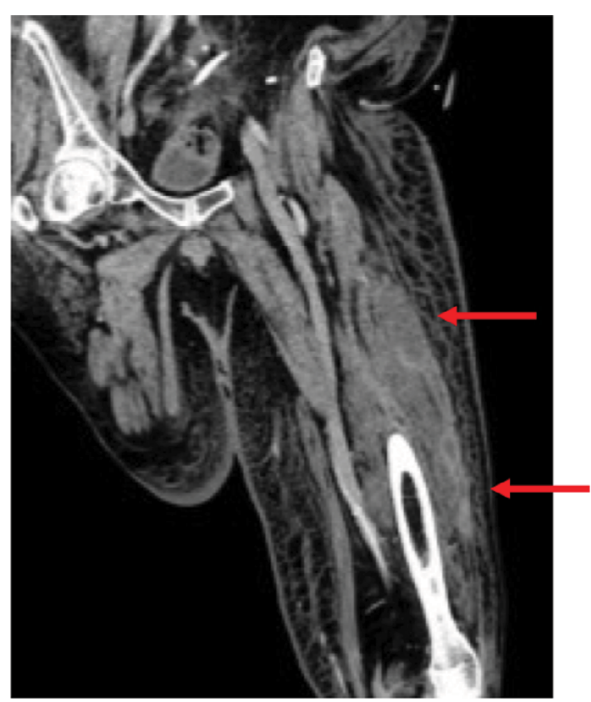

D

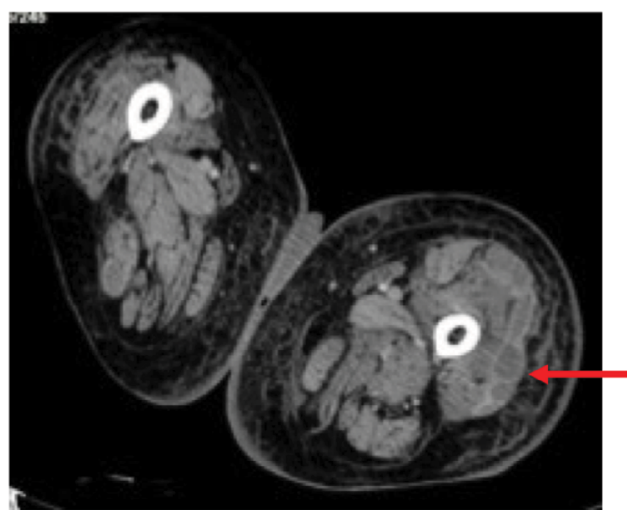

Figure 3 (A, B) CT-scan of left lower extremity, coronal sections showing abscess spanning the length of the vastus lateralis muscle. (C, D) Axial slices showing extent of abscesses. Red arrows indicate abscesses in vastus lateralis muscle.

literature, including both gram positives, negatives and even parasites and viruses. ${ }^{5}$ Gram positives are more frequently isolated, while $E$. coli is the most common gram negative. ${ }^{8}$ In our case, pyomyositis was caused by simultaneous $E$. coli and VRE infections, consistent with bowel flora as the initial source from perforated diverticulitis. In non-tropical areas, pyomyositis is uncommon, primarily affecting immunosuppressed, elderly patients. This disease is commonly associated with trauma and the immunodeficient state, which may include HIV, steroid use, diabetes mellitus, and neutropenia of any cause. ${ }^{1-2}$ Large muscles of the lower extremity are more commonly involved: the quadriceps, gluteal and iliopsoas muscles being the most common sites. ${ }^{2,4}$ Muscles remote to this location have been affected, including the sternocleidomastoid, erector spinae, and even muscles of the head and neck. ${ }^{4}$

Pyomyositis is divided into three stages. The first stage (invasive) typically lasts 2 weeks, with general symptoms of swelling, erythema, with or without pain or signs on radiologic imaging. The second stage (purulent) is when the disease is usually diagnosed, as in the case presented. Patients may have high fever, chills, sepsis with local tenderness, swelling, fluctuance over affected muscles, myalgia and inflammatory skin changes. In the third stage (late), the disease spreads systemically with worsening of local symptoms. Complications can occur at this stage, including rhabdomyolysis, hematologic spread of abscesses, arthritis and toxic shock syndrome from $S$. aureus. ${ }^{1,2,4}$

A high clinical suspicion must be maintained because imaging may not show discrete abscesses early in the course of the disease. Initial CT imaging in our patient showed gas in the gluteal muscles, which should have prompted further investigation. CT imaging in the first stage of the disease may only show swelling of the involved muscles. When abscesses are present, they appear as fluid collections with 
rim enhancement. MRI has been proven to be the most sensitive imaging modality in the diagnosis of this disease. It shows hyperintense signals on T2-weighted images and a hyperintense rim on enhanced T1-weighted images and peripheral enhancement after gadolinium administration. CT imaging and ultrasound can aid with diagnosis and treatment. ${ }^{4,7}$

Treatment of pyomyositis includes antimicrobial medical therapy and surgical or image-guided percutaneous drainage. If discovered in the invasive stage, antibiotic therapy alone is sufficient for treatment. ${ }^{6}$ Once the disease has progressed to the second or third stages, surgical or percutaneous drainage is necessary in conjunction with antibiotic therapy. Antibiotic therapy should be tailored to the speciation of cultures obtained from the abscesses. ${ }^{4,7}$

\section{Conclusion}

In this case, pyomyositis was secondary to bacteremia caused by purulent peritonitis from perforated diverticulitis evidenced by the isolation of bowel flora found intraoperatively in each intramuscular abscess. Several diagnostic challenges were present during this case. First, the diagnosis of diverticulitis was delayed, and the duration of perforation was unknown. The patient's immunosuppressed state obscured the usual clinical findings of diverticulitis, and the patient had a minimum of 5 days of purulent peritonitis before the disease was recognized and treated. Second, pyomyositis was not recognized, nor expected in the management of this disease. Several other diagnoses for extremity swelling and pain were explored before a CT scan was performed, revealing intramuscular abscesses. Once the abscesses were definitively treated with percutaneous drainage, the patient rapidly improved. Pyomyositis has not previously been described as a complication of complicated diverticulitis, and our experience shows that if unrecognized, can significantly prolong hospitalization and potentially lead to further complications.

\section{Acknowledgments}

The authors acknowledge and thank Richmond University Medical Center and the State University of New York Downstate Medical Center for their support in writing this report.

\section{Disclosure}

The authors report no conflicts of interest in this work.

\section{References}

1. Crum NF. Bacterial Pyomyositis in the United States. Am J Med. 2004;117(6):420-428.

2. Crum-Cianflone NF. Bacterial, fungal, parasitic and viral myositis. Clin Microbiol Rev. 2008;21(3):473-494.

3. Scriba J. Beitrag zur aetiologie der myositis acuta. Dtsch Z Chir. 1885;22:497-502.

4. Comegna L, Guidone PI, Prezioso G, et al. Pyomyositis is not only a tropical pathology: a case series. J Med Case Rep. 2006;10(1):372.

5. Levin MJ, Gardner P, Waldvogel FA. Pyomyositis: an unusual infection due to Staphylococcus aureus. N Engl J Med. 1971;284:196-198.

6. Ea HK, Zeller V, Chicheportiche V, Desplaces N, Ziza JM. Polybacterial pyomyositis following laparoscopic colectomy for complicated diverticulosis. Joint Bone Spine. 2007;74(6):653-655.

7. Drosos G. Pyomyositis. A literature review. Acta Orthop Belg. 2005;71(1):9-16

8. Khan S, Khan MA, Iqbal J, Saleem S. Pyomyositis, frequency and its common bacteria with their antibiotic sensitivity among children with highly suspected clinical features. Professional Med J. 2017;24(1):188-194.
International Journal of General Medicine

Publish your work in this journal

The International Journal of General Medicine is an international, peer-reviewed open-access journal that focuses on general and internal medicine, pathogenesis, epidemiology, diagnosis, monitoring and treatment protocols. The journal is characterized by the rapid reporting of reviews, original research and clinical studies across all disease areas.

\section{Dovepress}

The manuscript management system is completely online and includes a very quick and fair peer-review system, which is all easy to use. Visit http://www.dovepress.com/testimonials.php to read real quotes from published authors. 